\title{
Editorial
}

\section{Composite Nanoparticles}

\author{
Pietro Calandra, ${ }^{1}$ Valeria La Parola, ${ }^{2}$ Vincenzo Turco Liveri, ${ }^{3}$ \\ Elefterios Lidorikis, ${ }^{4}$ and Fabio Finocchi ${ }^{5}$ \\ ${ }^{1}$ CNR-IPCF, National Council of Research, Institute for Physico-Chemical Processes, Viale Ferdinando Stagno d'Alcontres, \\ 98158 Messina, Italy \\ ${ }^{2}$ Institute of Nanostructured Materials (ISMN-CNR UOS Palermo), Via Ugo La Malfa 153, 90146 Palermo, Italy \\ ${ }^{3}$ Dipartimento di Scienze e Tecnologie Biologiche Chimiche e Farmaceutiche (STEBICEF), Università Degli Studi di Palermo, \\ Viale delle Scienze, Edificio 17, 90128 Palermo, Italy \\ ${ }^{4}$ Department of Materials Science and Engineering, University of Ioannina, 45110 Ioannina, Greece \\ ${ }^{5}$ Institute of Nanoscience of Paris (INSP), CNRS and University Paris 6, Paris, France
}

Correspondence should be addressed to Pietro Calandra; calandra@me.cnr.it

Received 28 May 2013; Accepted 28 May 2013

Copyright (C) 2013 Pietro Calandra et al. This is an open access article distributed under the Creative Commons Attribution License, which permits unrestricted use, distribution, and reproduction in any medium, provided the original work is properly cited.

Composite nanoparticles are advanced materials having recently gained increasing attention due to their scientific and technological importance. They find a wide variety of applications such as catalysts with huge activity and specificity, metal semiconductor junctions, optical sensors, and modifiers of polymeric films for packaging.

From a scientific point of view the composition and the atomic order of the aggregates, in addition to size, are pivotal factors in determining their properties and functionalities, while the nanoscale regime confers to them structural and electronic degrees of freedom which are inaccessible to bulk materials.

The first indispensable stage to develop novel nanotechnologies is the preparation of tailored composite nanostructures. Virtually all the possible physicochemical phenomena have been employed to reach this objective allowing to set up a huge number of protocols each one with its specific advantages and disadvantages. The aspects of novel preparation methods are addressed in two manuscripts.

S. Bagheri et al. describe a novel synthesis of anatase titanium dioxide nanoparticles using egg white solution via solgel method and characterized them through a high number of techniques. The main advantage of using egg white proteins as a gelling agent is that it can provide long-term stability for nanoparticles by preventing particles agglomeration. They demonstrated that egg white solution is a reliable and cheap green gelling agent that can be used as a matrix in the sol-gel method to synthesise tiny size TNPs.

V. Ovchinnikov and A. Shevchenko describe a costeffective fabrication of random noble-metal nanostructures with a feature size of the order of $10 \mathrm{~nm}$ on a large-area dielectric substrate. The technique allows to create metal nanoislands on a nanopatterned dielectric template with an enhanced adhesion between the metal and the dielectric. The use of the adhesion layer (that makes the structures stable) is important in view of variety of optical and other potential applications of the structures. The results of their work can be of interest in regard to the development of new approaches to self-organization based nanofabrication of extremely small metal and metal-dielectric nanostructures on large-area substrates.

Due to their peculiarities, the production of nanomaterials is a promising strategy to fuel novel technological applications. Two manuscripts, highlighting enhanced properties for applicative purposes, are good examples of the potentialities of these materials.

M. C. Carrera et al. studied the permeation of $\mathrm{CO}_{2}$ in films of high density polyethylene (HDPE) and organoclay modified with polyvinylalcohol $\left(\mathrm{MMT}_{\mathrm{HDTMA} / \mathrm{PVA}}\right)$ obtained from melt blending. They showed that the incorporation of the modified organoclay generated an exfoliated nanocomposite structure with a significant effect on the barrier 
properties of HDPE. Notably the mechanical properties remain equal to those of pure polyethylene, but with an increase in barrier properties to $\mathrm{CO}_{2}$ allowing to obtain nanocomposites of HDPE competitively used in packaging.

Q. X. Li et al. prepared a novel $\mathrm{Pd} / \mathrm{C}$ catalyst with enhanced performance for direct ethanol fuel cell. They characterized the catalysts by XRD and TEM showing that they are spherical and homogeneously dispersed on carbon.

Finally, the theoretical/computational approach has been faced by P. Shabanzadeh et al. In their investigation, they applied the accuracy of artificial neural network training algorithm to study the effects of different parameters on the synthesis of Ag nanoparticles performed in the interlamellar space of montmorillonite (MMT) by chemical reduction technique. Using the best performing artificial neural network, they predicted the optimum conditions of $\mathrm{AgNO}_{3}$ concentration, MMT d-spacing, and reaction temperature.

By compiling these papers, we hope to enrich our readers and researchers with respect to the most recent progresses in the field of nanoscale multicomponent particles.

Pietro Calandra Valeria La Parola

Vincenzo Turco Liveri Elefterios Lidorikis

Fabio Finocchi 

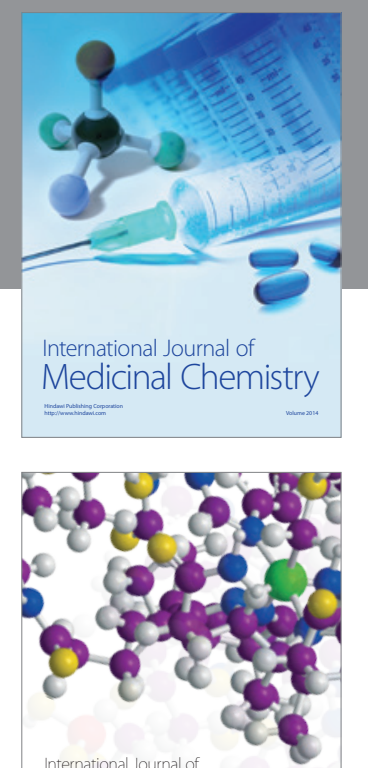

\section{Carbohydrate} Chemistry

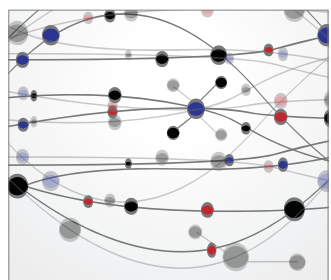

The Scientific World Journal
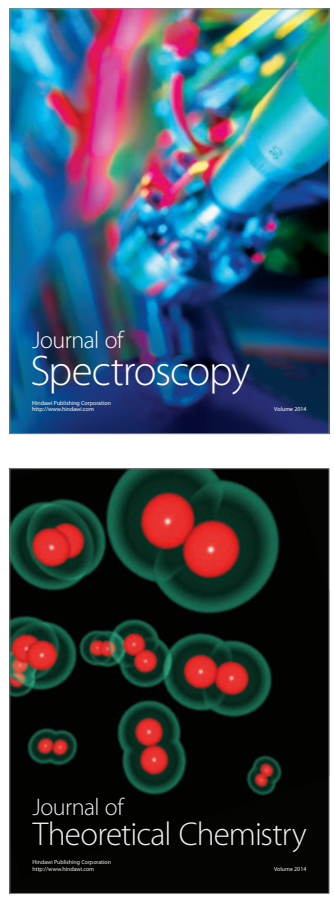
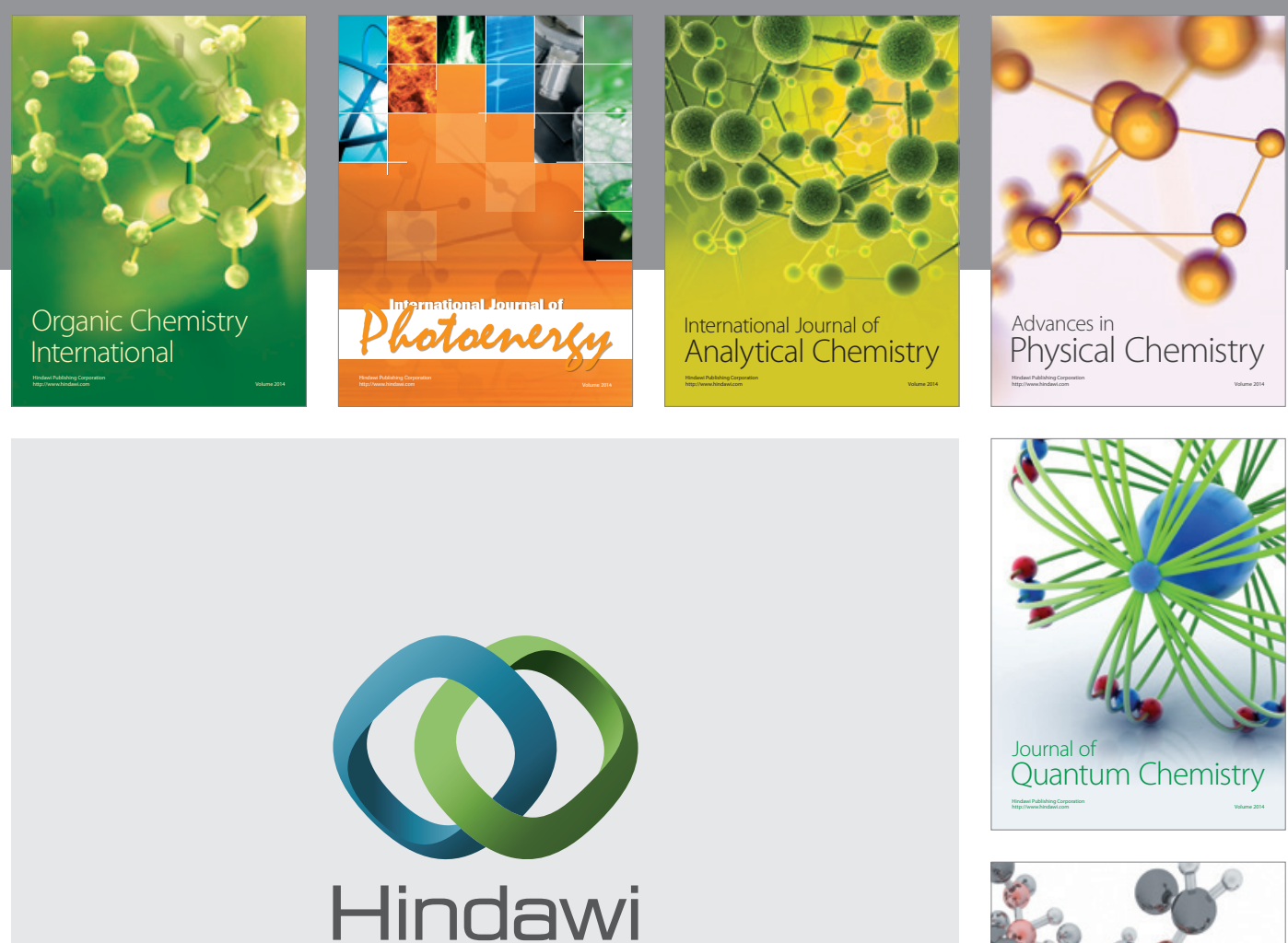

Submit your manuscripts at

http://www.hindawi.com

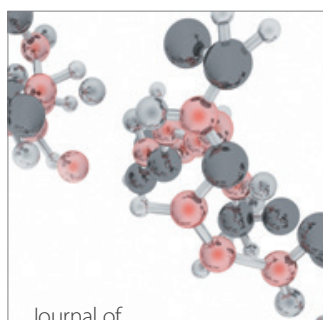

Analytical Methods

in Chemistry

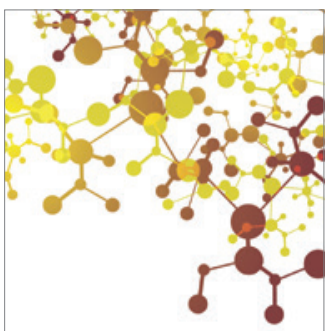

Journal of

Applied Chemistry

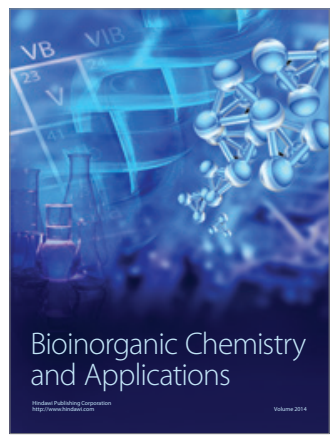

Inorganic Chemistry
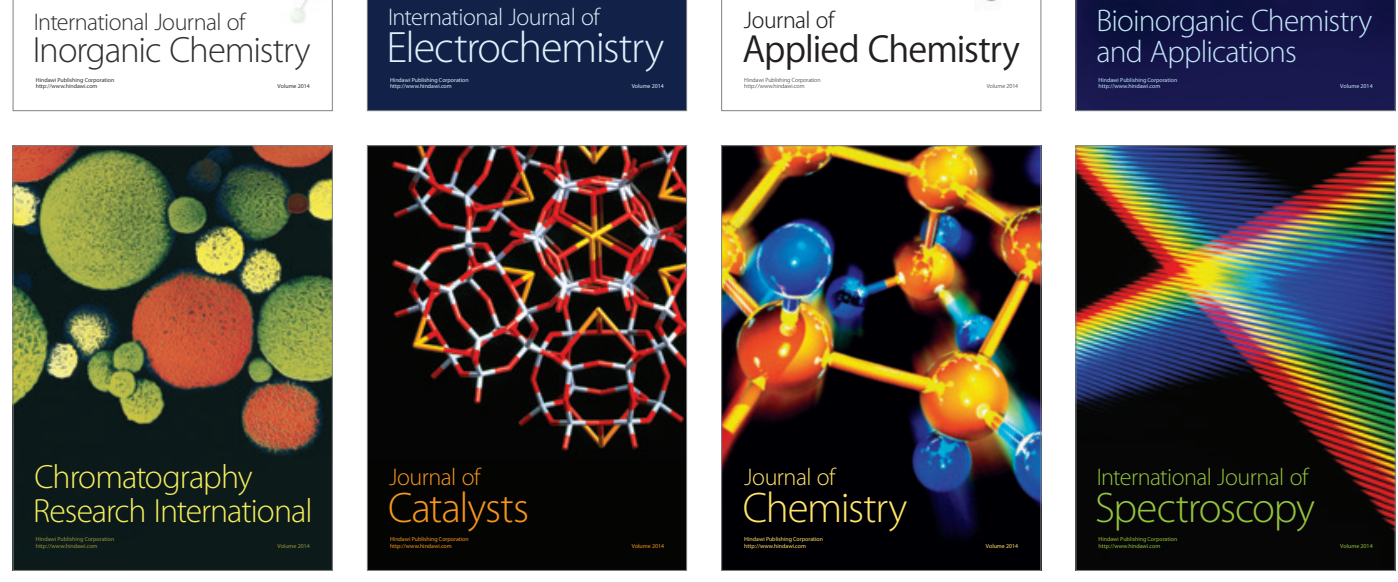